\title{
Facial Respirator Shape Analysis using 3D Anthropometric Data
}

Riley Fenlon

\author{
National Institute of Standards and Technology
} Technology Administration, U.S. Department of Commerce 
NISTIR 7460

\title{
Facial Respirator Shape Analysis using 3D Anthropometric Data
}

\author{
Riley Fenlon \\ Information Technology Laboratory \\ Information Access Division
}

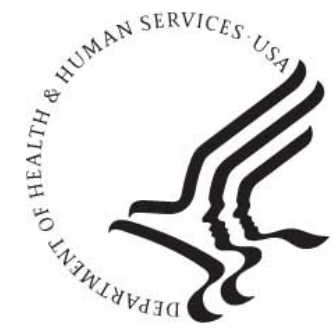

U.S. Department of Health and Human Services Mike Leavitt, Secretary

Centers for Disease Control and Prevention Julie Louise Gerberding, Director

National Institute of Occupational Safety and Health John Howard, Director
National Institute of Standards and Technology William Jeffrey, Director 


\begin{abstract}
For firefighters and first response crews, the facial respirator is the first and last defense against hazardous and potentially harmful airborne agents. That defense is crippled if the seal of the respirator sprouts a leak, exposing the wearer to the dangers of the surrounding environment. Due to the inherent limitations of traditional anthropometric measures in modeling the nuanced and complex human face, fit testing standards for respirators remain imprecise. A recent review of the National Institute for Occupational Safety and Health (NIOSH) anthropometric survey called for 3D facial data to try and remove some of this uncertainty.

Using the Civilian American and European Surface Anthropometry Resource (CAESAR) database finished in 2000, 3D facial models were created to test the rigid body fit of a half-mask respirator across different demographic groups. A good fit, in this study, is defined by a relatively small total distance between the face and the landmark points of the mask. Employing a best fit algorithm, the average face generated for Asians surprisingly accrued the least amount of gap while the male face had the greatest gap. When the landmark points of the mask were superimposed on a contour plot of the standard deviation in the face of each group, the plot revealed that the mask extended into areas of high facial variance. Upon analysis of the gap distance at each landmark, it was found that the sides of the mask were the areas with greatest separation from the face. Further investigation of wider than average faces confirmed little to no correlation between head breadth and total gap distance.
\end{abstract}




\section{ACKNOWLEDGEMENTS}

The funding and support for this report was made possible by the Systems Integration for Manufacturing Applications (SIMA) and the Summer Undergraduate Research Fellowship (SURF) at the National Institute of Standards and Technology (NIST). The author would like to gratefully acknowledge the assistance of his advisor, Afzal Godil, who provided guidance and insight at every juncture. The author would also like to acknowledge the help of Kathryn Butler in providing the mask used in the analysis and reviewing this paper. Thanks are also given to Damien Bertot and Charles McLean for performing the $3 \mathrm{D}$ scan of the mask.

\section{DISCLAIMER}

Commercial products are cited in reference to the gap analysis procedures. The identification of such products is neither a recommendation nor endorsement by the National Institute of Standards and Technology, but is included for the purpose of experimental reproducibility. 


\section{TABLE OF CONTENTS}

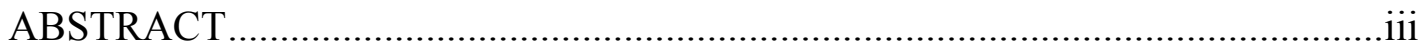

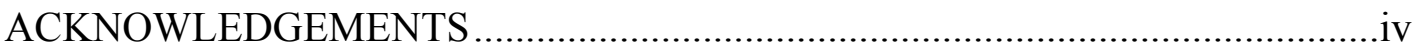

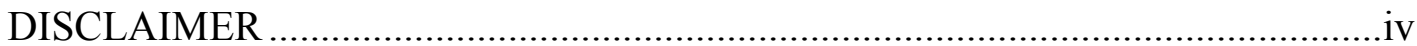

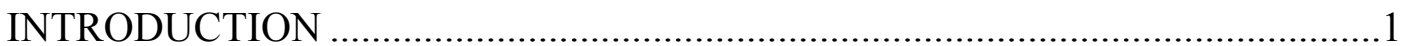

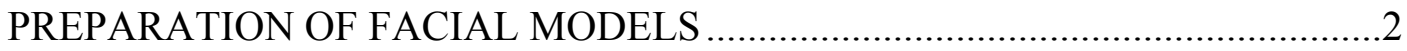

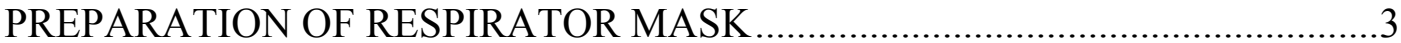

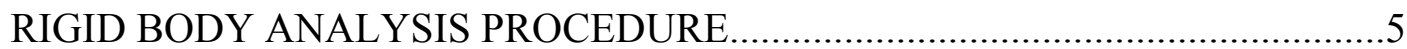

QUANTITATIVE AND QUALITATIVE GAP ANALYSIS RESULTS .................8

Results: Total Gap by Demographic Analysis...............................................

Results: Analysis of Gap at Parts of the Respirator .......................................9

Results: Correlation between Head Breadth and Fit......................................10

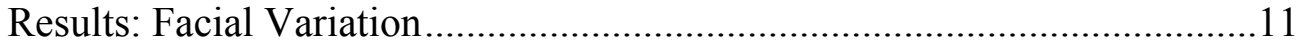

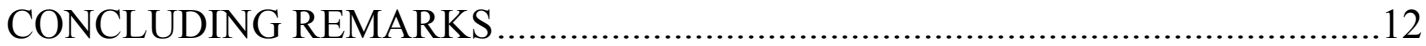

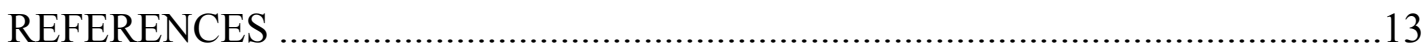




\section{INTRODUCTION}

For firefighters and first response crews, the facial respirator is the first and last defense against hazardous and potentially harmful airborne agents. At the crux of this defense is the seal of the respirator, which prevents harmful gases and particulates from gaining access to the wearer's respiratory system. Respirators must be tough enough to withstand stress from the often laborious (or physically taxing) working conditions, yet malleable enough to maintain a proper seal during elevated breathing rates and facial motion from speaking - a difficult line to balance for manufacturers. If a gap or leak occurs in the seal, then the rest of the respiratory protective equipment is essentially rendered useless, leaving the wearer vulnerable to airborne threats. Although the importance of respirators is universally recognized, the fit test panels to certify respirators remain imprecise due to their dependence on an antiquated source survey.

The Los Alamos National Laboratory (LANL) fit test panel developed in the 1970's is based on an anthropometric survey conducted in 1967 of Air Force personnel and still the standard for today's respirator fit tests. Subjects of the survey were generally young, physically fit individuals with limited diversity - a sample not representative of today's civilian population. Considering the age of the survey and the dramatic change in the composition of the U.S. population, the National Institute for Occupational Safety and Health (NIOSH) conducted a new survey in 2001 entitled the NIOSH Head-and-Face Anthropometric Survey of U.S. Respirator Users to produce a more accurate picture of the civilian workforce. Subsequent analysis of the survey revealed that the LANL panels were in fact not representative of most respirator users in the U.S. Out of the 3997 respirator users in the survey, $15.3 \%$ of them were outside of the LANL fit test panel.

Although the fit test panel is in the process of being updated, the core of these fit tests is still traditional anthropometric measures, which oversimplify the nuances and complexity of the human face. The contours, textures, and flexibility that make each face unique are largely ignored when using traditional measures with calipers. NIOSH understood this and had 3D facial scans initially implemented with the survey. However, the surveyors noticed discrepancies between traditional measures and the values extrapolated from the 3D scans. As a result, the 3D scans were not included in the final version of the revised NIOSH fit test panel, despite being essential to a full picture of the face.

Previous studies in facial respirators have used 3D spatial landmarks to model the face, but this again is a large simplification of the geometry of the face. Recognizing this shortcoming, this study used the Civilian American and European Surface Anthropometry Resource (CAESAR) database finished a year prior to the NIOSH study to create 3D facial models to test the rigid body fit of a half-mask respirator across different demographic groups. It has been shown that a respirator offers its best protection when the seal fits a face properly. In light of this, a lower rigid body total gap between the face and the respirator landmarks is assumed to correlate to a better fit. Results from the preliminary total gap analysis led to an additional study into the correlation of head breadth and fit. 


\section{PREPARATION OF FACIAL MODELS}

Before any investigation into the face can begin, 3D scan data must be uncompressed into a readable form and then transformed into a universal orientation for analysis. All of the calculations and algorithms were coded and compiled using Java based MATLAB 7.3.0 (R2006). The CAESAR scans were saved in a .ply file format and were converted into two files: one containing the Cartesian coordinates of the points and the corresponding triangular connectivity and the other containing color information.

With the scan points in a readable form, the full body needed to be trimmed and oriented uniformly to allow for deviations and average values to be extrapolated in a reasonably accurate manner. Varying heights of subjects and the inconsistent tilt of the face during the scan scattered the desired facial grid points in Cartesian space, requiring a series of affine transformations to bring the faces to the same location. Scaling was not performed on the faces because both size and shape of the face are relevant to a fit test of a mask. To achieve uniform agreement of the faces, the sellion landmark was placed at the origin $(0,0,0)$ with the length of the face along the y-axis, the width of the face along the x-axis, and the depth of the face along the z-axis. Fig.1 shows the seated full body scan of a subject with all of the landmark points labeled.

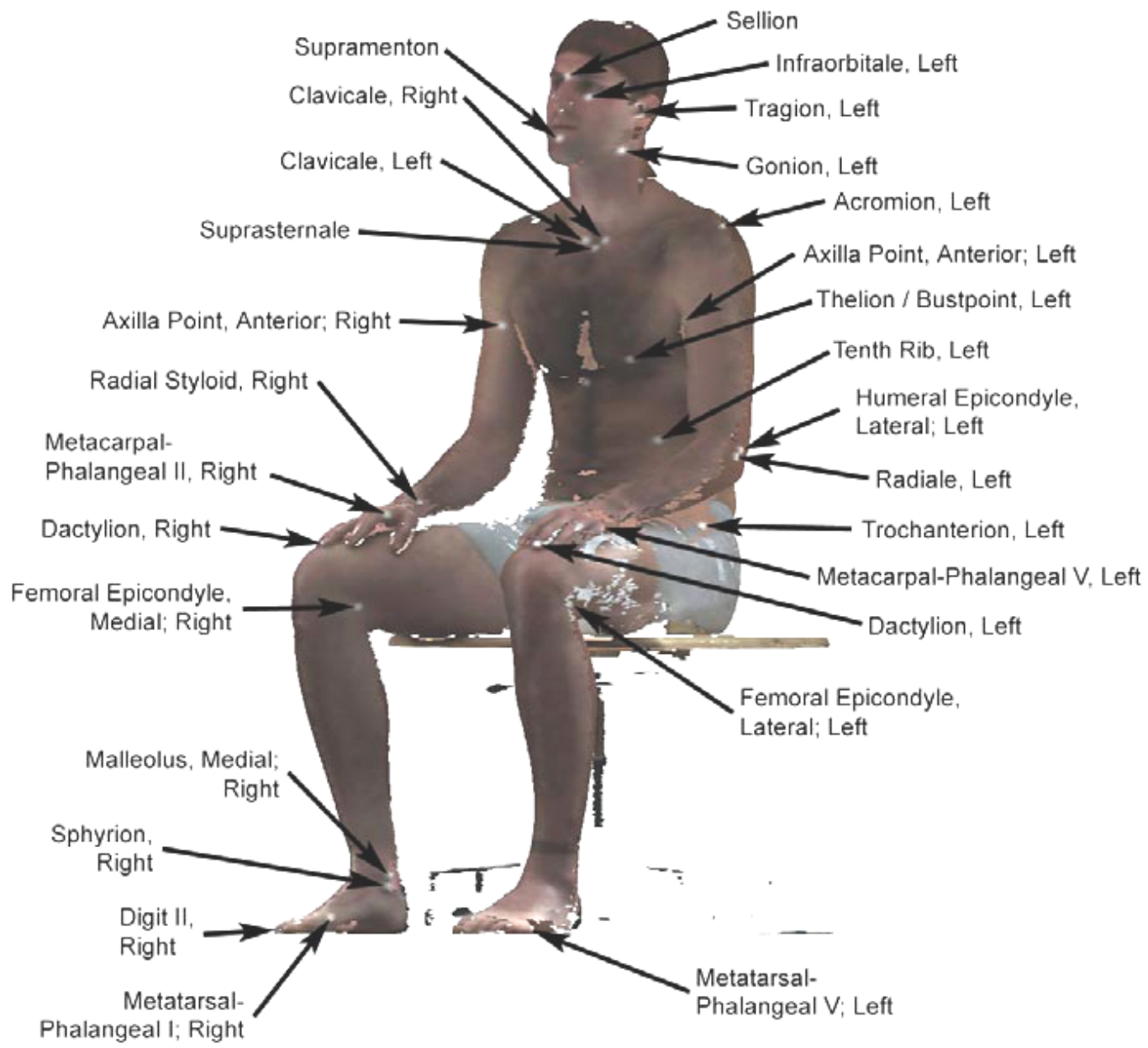

Fig. 1: Seated CAESAR subject with landmark points labeled 
Not needing any points below the neck, the points below right and left clavicle landmarks were immediately cut to make subsequent transformations quicker. Then, using a common 3D translation matrix, the head was translated so that the sellion landmark was at the origin. A rotation around the $\mathrm{z}$-axis aligned the supramenton and sellion along the $\mathrm{x}=0$ plane. Then, a rotation around the $\mathrm{x}$-axis aligned the supramenton directly below the sellion on the y-axis. After that, a rotation along the y-axis adjusted the left and right infraorbitale to give them the same depth. Fig. 2 b) illustrates the data manipulation up until this point. Finally, the back of the head was cut off using the left and right tragion landmark as the threshold. MATLAB interpolated a uniform mesh overtop the face and estimated the appropriate $\mathrm{z}$ value from the surrounding grid points. The final product is a 3D surface of the front of a face looking directly up the z-axis. Fig. 2 c) shows the final interpolated surface of a subject's face.

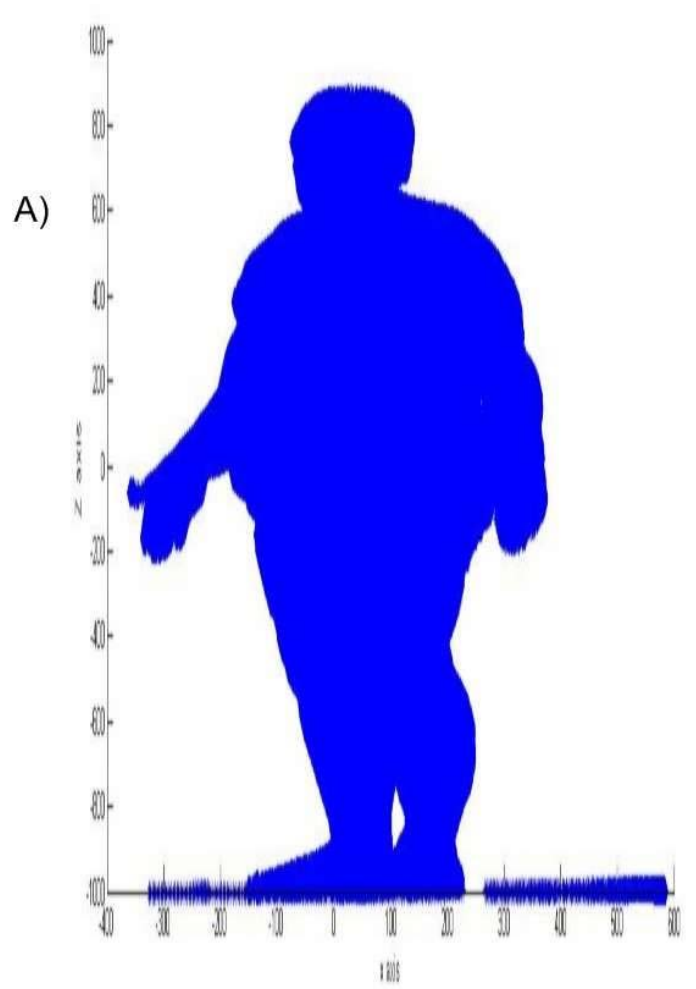

B)

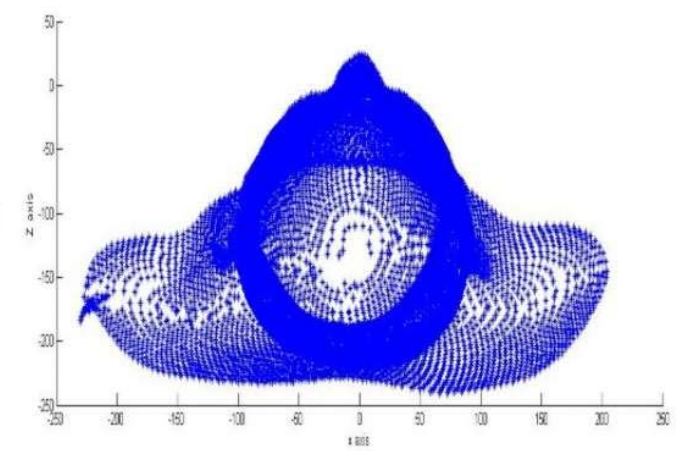

C)

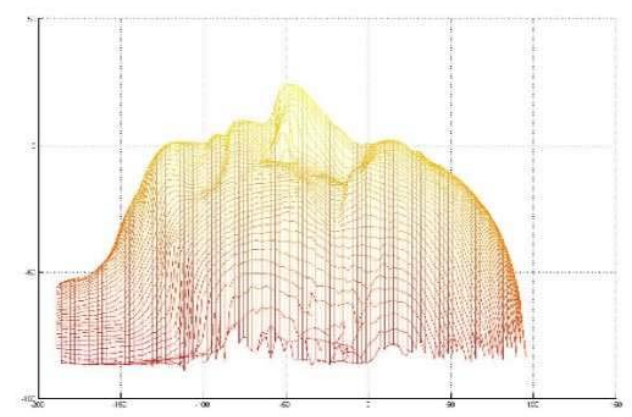

Fig. 2 a) plot of all original, unchanged scan points for a full body scan in the CAESAR database

b) view from top of a correctly oriented head of grid points

c) plot of facial surface looking up $\mathrm{z}$ axis after cutting and interpolation

\section{PREPARATION OF THE RESPIRATOR MASK}

The mask used in the study is a medium (comes in medium and large sizes) half face air-purifying respirator (APR). Firefighters typically use a full face self-contained breathing apparatus (SCBA) in immediately dangerous to life or health (IDLH) environments, but the half mask provides insight around the chin and nose-areas where leaks are very common. Further, half mask respirators are similar to the nosepiece within 
the full face SCBA used by firefighters. Therefore, a half mask respirator was chosen as an effective model despite the fact that it is not directly used by firefighters.

The 3D scan data of the respirator mask was gathered using the Konica Minolta Vivid $9 \mathrm{i}$ at the Manufacturing Engineering Laboratory at NIST. Rendering and data writing were performed using Geomagic software. Fig. 3 a) displays the rendering of the 3D scan data of the respirator mask in Geomagic. As the respirator did not come with any landmark points, four points were arbitrarily chosen; northernmost, westernmost, easternmost, and southernmost. These landmarks were chosen to mimic the four landmarks used in orientation of the face with the northernmost acting as the sellion, the southernmost acting as the supramenton, and the westernmost and easternmost acting as the left and right infraorbitale respectively. Orientation can be seen in Fig. 3 b).

A)

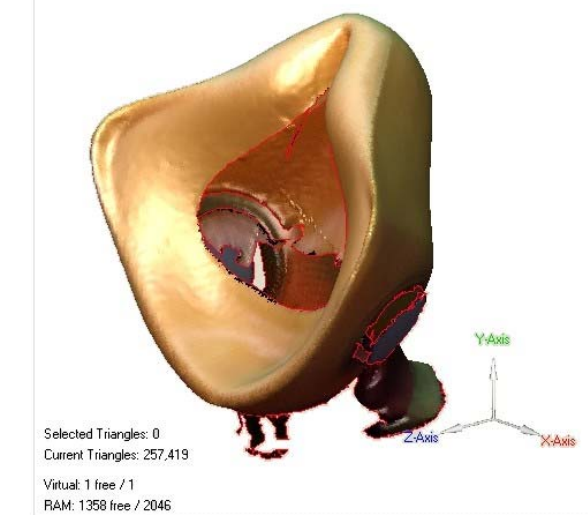

B)

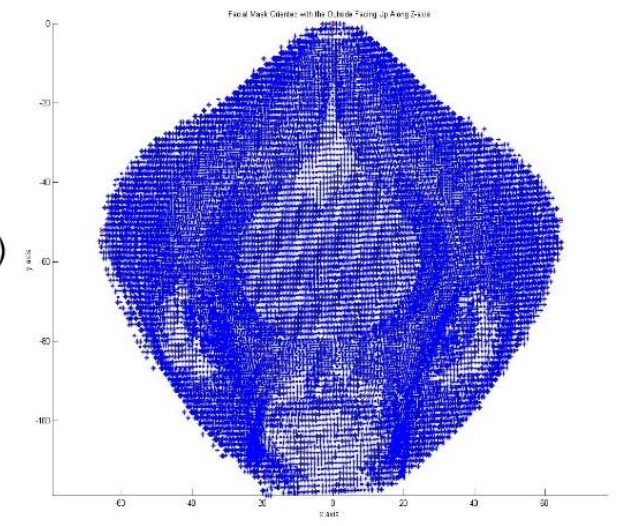

C)

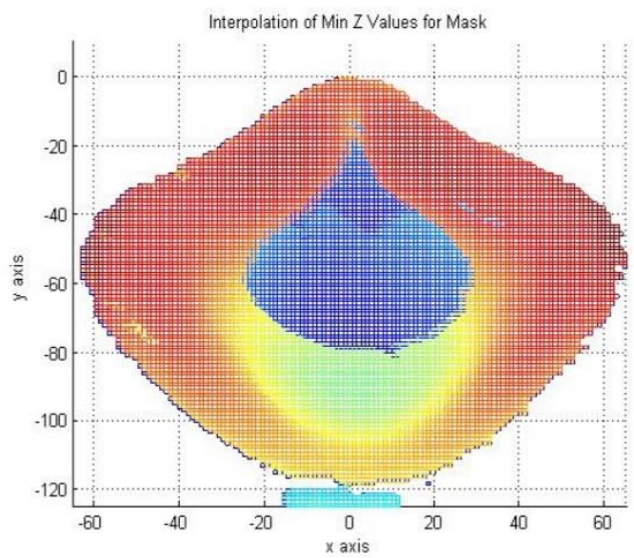

D)

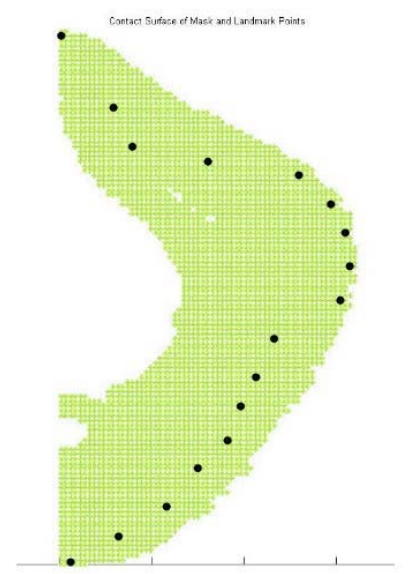

Fig. 3 a) rendering of the mask performed in Geomagic

b) plot of the grid points of the mask after orientation

c) interpolated surface of the contact area of the mask

d) chosen landmark points superimposed on top of contact surface

The mask was oriented using the same process performed on the face-the northernmost point was translated to the origin with the southernmost point directly below it on the y-axis. However, the mask was not as easy to interpolate. If multiple $z$ values exist at a given $\mathrm{x}$ and $\mathrm{y}$ coordinate, then the interpolation function uses the average of the $\mathrm{z}$ values in creating the surface. Only the surface of the mask that comes into contact with the face is of interest in this study. As a result, the outside of the mask was 
neglected and the minimum $\mathrm{z}$ value for each $\mathrm{x}$ and $\mathrm{y}$ coordinate was used for interpolation. Fig. 3 c) displays the contact area of the mask that was used in analysis. In the interest of saving time while performing gap analysis, perfect symmetry of the mask was assumed and only the right side was considered. Seventeen landmark points were chosen on the final surface. These landmarks were the minimum $\mathrm{z}$ values for seventeen evenly spaced rows from the top to the bottom of the contact surface because it was believed that these minimum $\mathrm{z}$ values were the most likely to be the areas of first contact with the face. Fig 3 d) displays the contact surface with the landmark points superimposed on top. These seventeen landmark points would represent the entire mask in subsequent best fit and gap analysis procedures. In general, the mask landmarks near the center of the face represented the mask well, but the points near the side of the face were generally too far - a discrepancy created most likely because the sides of the mask were intended to be pulled to the side of the face by the respirator harness. 


\section{RIGID BODY ANALYSIS PROCEDURE}

Of particular interest to facial anthropometry is differences based on race and gender. Understanding the inherent differences in facial structure between races and genders, respirator manufacturers tailor their products to better fit the population subset that is most likely to use their product. This study used broader ethnic classifications than the ones provided by the CAESAR database because the American survey size consisted of only 2400 subjects. The final groups considered in the study are: Asian/ Pacific Islander, White, Black, Hispanic, Other/Mixed, Male, and Female. Further classification of the subjects produced sets that were too small for proper analysis.

With the demographic separations drawn, the average face for each group was generated. A simple calculation of the average $\mathrm{z}$ value at each $\mathrm{x}$ and $\mathrm{y}$ coordinate in the mesh for every subject would not suffice because the result is an amorphous face with poorly defined features that do not resemble that of a person. As a result, the traditional anthropometric measures provided in the database were used to identify the subjects that were the closest to the "average face" for each group. The subjects that were within the $40^{\text {th }}$ and $60^{\text {th }}$ percentile in head breadth and $40^{\text {th }}$ and $60^{\text {th }}$ percentile in face length for each group were considered to be representative of the "average face". Then, using this smaller representative group, the average $\mathrm{z}$ value at each $\mathrm{x}$ and $\mathrm{y}$ coordinate was calculated; the result was an average face with much more human features. When the time came to investigate the correlation of head breadth and fit, the thinner face for each demographic group was generated from subjects in the $5^{\text {th }}$ to $25^{\text {th }}$ percentile in head breadth and $40^{\text {th }}$ to $50^{\text {th }}$ percentile in face length and the wider face for each demographic was generated from subjects in the $75^{\text {th }}$ to $95^{\text {th }}$ percentile for head breadth and $40^{\text {th }}$ to $50^{\text {th }}$ percentile in face length. Fig. 4 shows the side by side comparison of the wide face, average face, and skinny face.
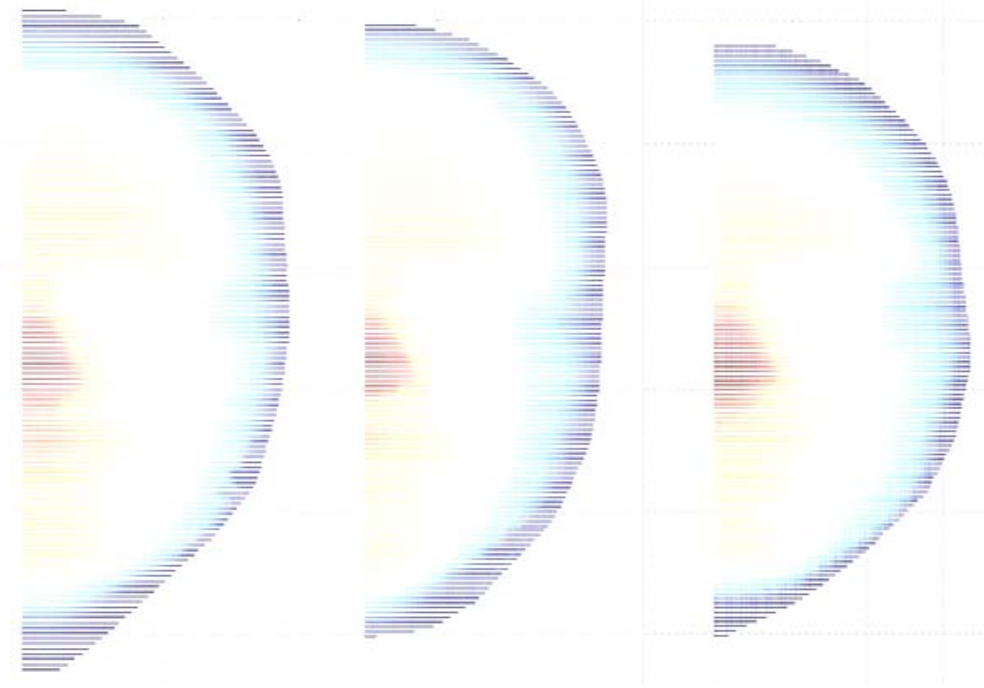

Fig. 4 shows the male wider face (left), average face (center), and skinnier face (right)

With the face for each group generated and the landmark points of the mask chosen, the final step was to create a best fit algorithm to define a unique best fit for each face. This study assumed a rigid body analysis, which neglected flexing or deformation of the face or mask. Under these constraints, the best fit was defined as the arrangement of the 
landmark points that accrued the smallest total distance between the face and the landmark points of the mask. The gap at each landmark was defined as the minimum Cartesian distance $\left(\mathrm{d}=\sqrt{ }\left(\left(\mathrm{x}_{1}-\mathrm{x}_{2}\right)^{2}+\left(\mathrm{y}_{1}-\mathrm{y}_{2}\right)^{2}+\left(\mathrm{z}_{1}-\mathrm{z}_{2}\right)^{2}\right)\right)$ between the mask landmark and the face surface. Fig. 5 illustrates the results of the minimum distance function by plotting the landmark point and its corresponding closest point on the face.

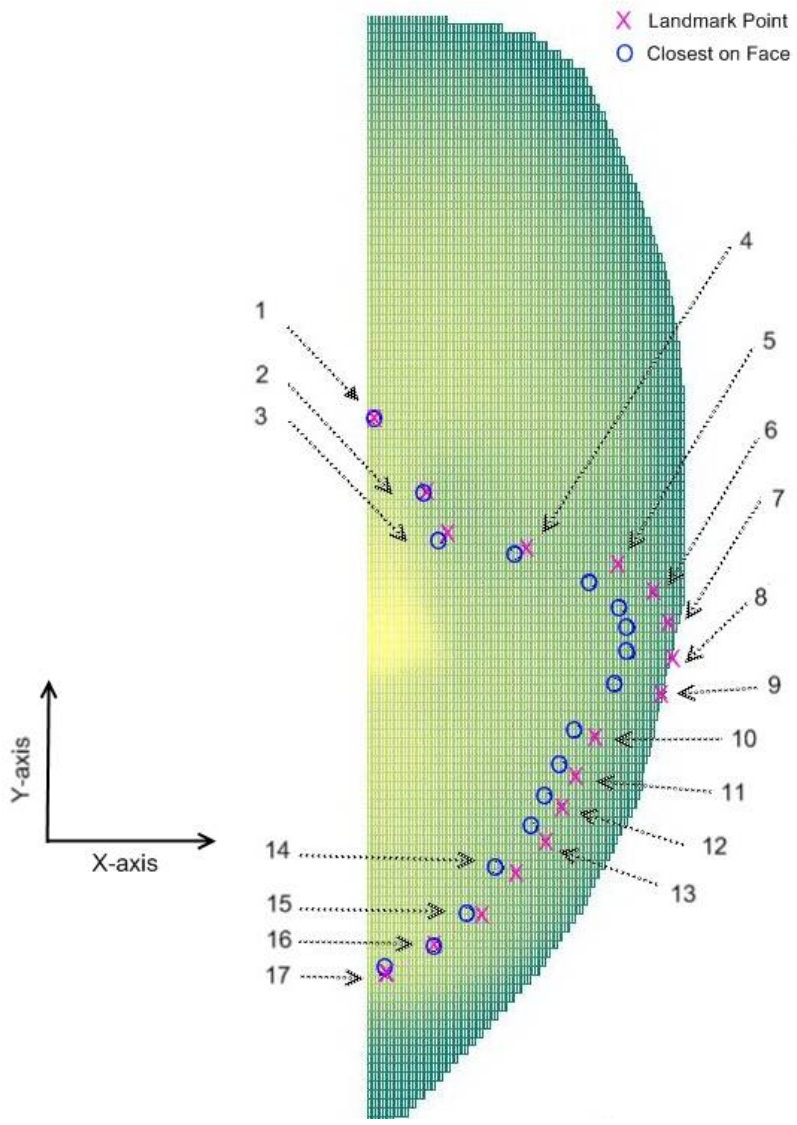

Fig. 5 is a diagram illustrating the minimum distance algorithm. The numbered mask landmarks are identified by a magenta $\mathrm{X}$ and the corresponding nearest points on the face are blue circles.

The best fit algorithm developed was an iterative process consisting of three affine transformations: a translation along the $\mathrm{z}$ axis brought all of the landmark points just outside the surface of the face; a translation along the y axis moved the mask up and down the face around the bridge of the nose; and a rotation around the $\mathrm{x}$ axis tilted the mask away and toward the bridge of the nose. If, during the course of the iteration, the program found a transformation that produced a lower total gap than the current position of the landmarks, then the current position of the landmarks would automatically update itself. Appropriate transformation limits were set to prevent the mask from finding a best fit that was unrealistic. For instance, a best fit that placed the top of the mask on the forehead of a face would not be allowed under the limits. At no time would a transformation that placed a landmark point inside the surface of the face be accepted as the best fit. After each iteration, the transformations became finer until the algorithm converged or reached 10 iterations, whichever came first. 


\section{QUANTITATIVE AND QUALITATIVE GAP ANALYSIS RESULTS}

\section{Results: Total Gap by Demographic Analysis}

Running the best fit algorithm on the average face for each demographic yielded total gap data as shown in Fig. 6. As can be seen from the graph, the Asian/ Pacific Islander group had by far the smallest total gap with $78.32 \mathrm{~mm}$; the next closest demographic was the average Black face with $101.22 \mathrm{~mm}$. Whites, Hispanics, Other/Mixed, and females finished in the middle of the pack, but the demographic with the worst total gap was the average male face with $133.00 \mathrm{~mm}$. When looking at labor force statistics for the United States, the results appear counterintuitive. As of June 2007, Whites have the largest labor force by race with 125.9 million compared to Asians with a mere 7.1 million in the labor force. Further, males have 83.0 million people 16 years or over in the labor force while females have 71.2 million people 16 years or over as of June 2007. Asians, as a relatively small segment of the U.S. labor force, appear to have garnered the least total gap across all the races. One possible explanation for this peculiarity is that the medium sized respirator was designed to better fit smaller faces. Traditional measures provided by the CAESAR database seem to support this claim because Asians have the smallest median head circumference and males have the largest median head circumference.

\section{Best Fit Total Gap Distance}

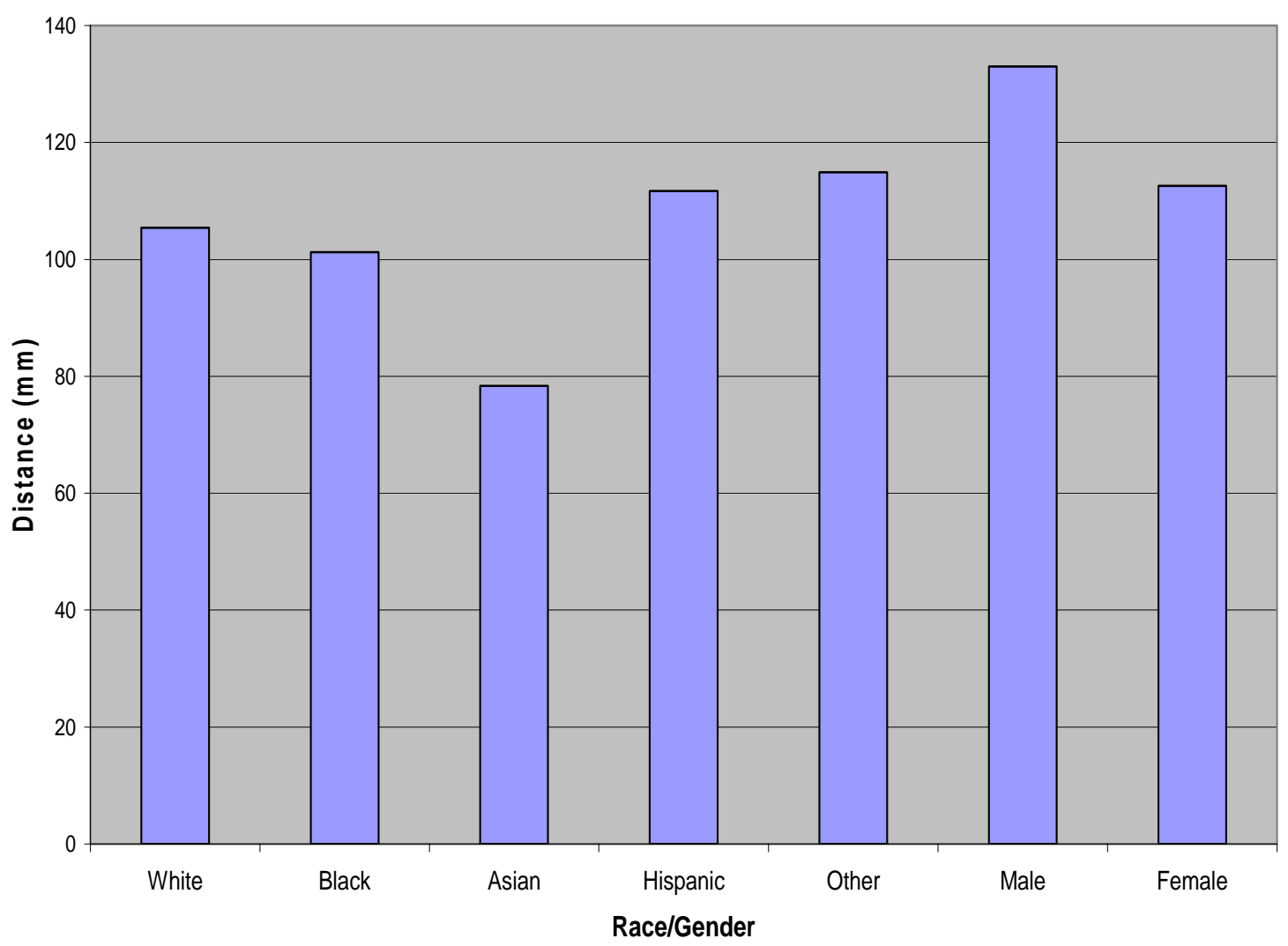

Fig. 6 shows the total gap for the respirator best fit on the average face for each demographic 


\section{Results: Analysis of Gaps at Parts of the Respirator}

In light of this oddity in the data, a more detailed analysis of the parts of the mask was performed. Rather than considering the total gap for each demographic, the gap at each landmark was recorded. As can be seen from Fig. 7, landmarks 5-9 generate the greatest gap for the faces with values reaching almost $16 \mathrm{~mm}$. All of the other landmark points accrue a negligible gap in comparison with values barely exceeding 9 millimeters. The mask landmarks 5-9 appear near the side of the face by the steep contours of the cheeks as can be seen in Fig. 5. The appearance of this could be attributed to the selection of the landmark points on the mask. If landmarks 5-9 were chosen to be closer to the center of the face as indicated by the blue circles on the face in Fig. 5, then the gap would logically be significantly smaller. Regardless of the cause of the trend, the results of the parts analysis compelled an investigation into the correlation between head breadth and fit.

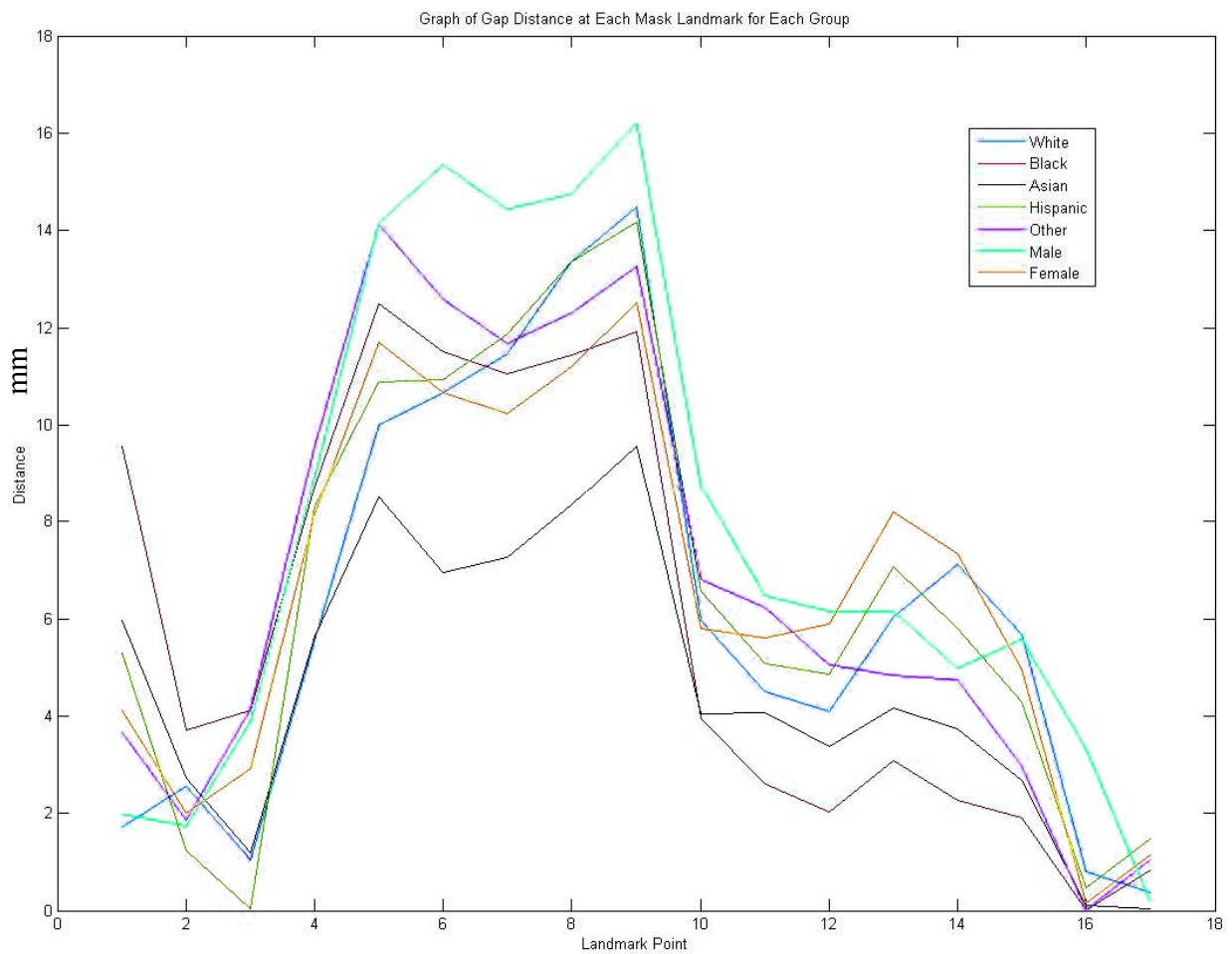

Fig. 7 is a plot of the gap distance at each landmark of the mask for the average face in each demographic group. Notice the large gaps around landmarks 5 through 9. Distance is in millimeters. 


\section{Results: Correlation between Head Breadth and Fit}

The wider face for each demographic was taken from the average $\mathrm{z}$ values of the subjects inside the $40^{\text {th }}$ to $60^{\text {th }}$ percentile in face length and $75^{\text {th }}$ to $95^{\text {th }}$ percentile in head breadth; the skinnier face for each demographic was the average $\mathrm{z}$ values of the subjects inside the $40^{\text {th }}$ to $60^{\text {th }}$ percentile in face length and the $5^{\text {th }}$ to $25^{\text {th }}$ percentile in head breadth. It was hypothesized that a wider face would compliment the relatively flat contours of the respirator and fill the void around landmarks 5-9, resulting in a lower total gap. In the case of males, this relationship proved to be quite true with each wider face bringer a lower total gap. However, the reverse trend appeared in the Other face with gap actually increasing as width increased. Across the board, Fig. 8 shows the apparent dissimilarity between head breadth and total gap, further showing the difficulty of traditional anthropometric measures to predict the fit of a respirator.

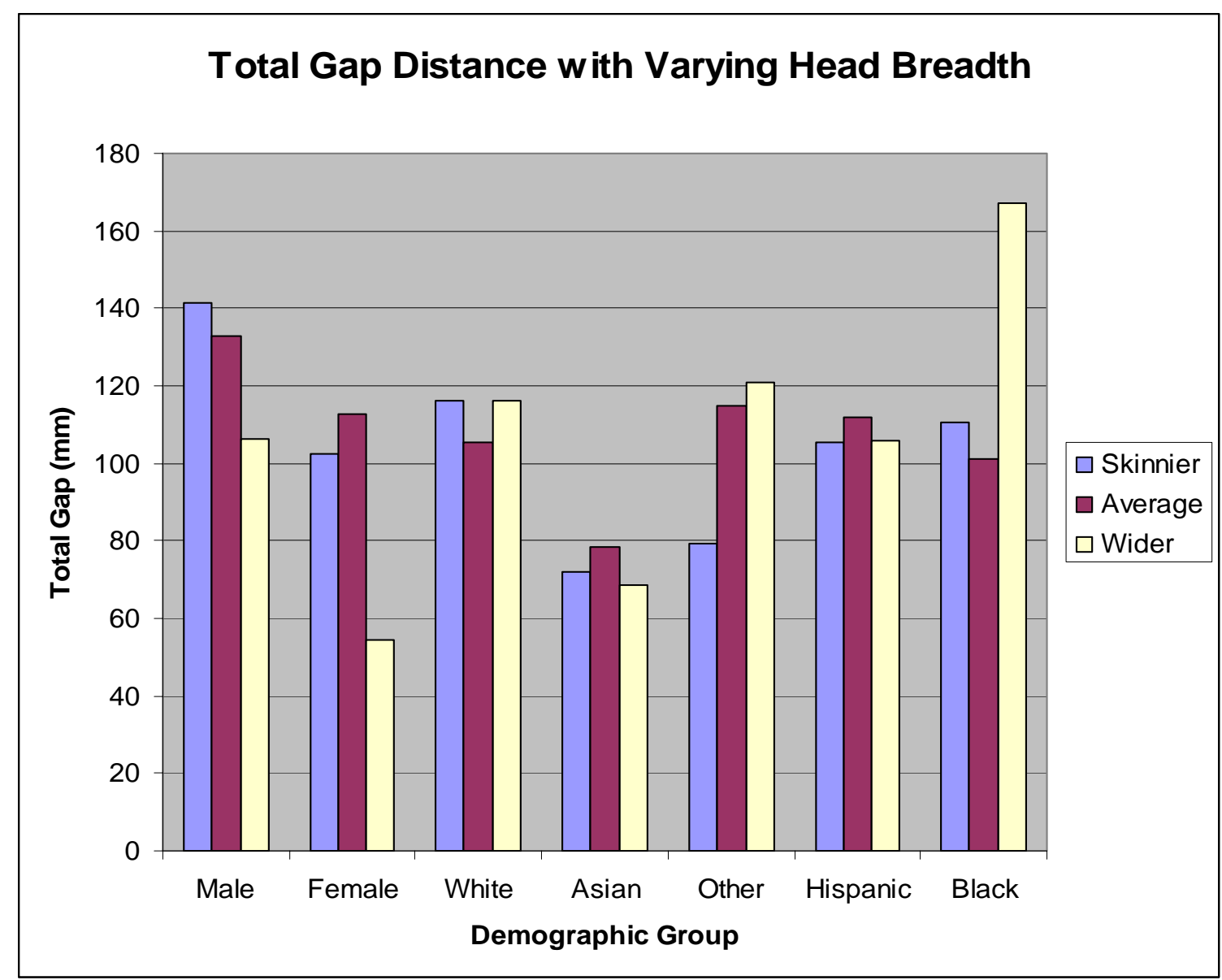

Fig. 8 is a plot of the total gap distance across all demographics with a varying head breadth

Despite the lack of a concrete correlation between head breadth and fit across demographic groups, this inquiry supported some trends that arose in the initial investigation; females continued to have a lower total gap than males, Asians continued to be near the bottom, and Whites consistently finished near the top. The fact that these trends continued in spite of the aforementioned labor force statistics is interesting. Once 
again this trend could be explained by the selection of the mask landmark points and the target market of the medium sized mask.

\section{Results: Facial Variation}

One final study involved the qualitative observation in the variation of the face for each demographic group. A contour plot of the standard deviation in the $\mathrm{z}$ value at each $\mathrm{x}$ and $\mathrm{y}$ coordinate created a visualization of which areas in the face for each demographic have the most variation. Due to the way all of the faces were oriented, considerable bias exists at the bridge of the nose and the chin where variations are lowest. During orientation, all of the sellion landmarks of the face are brought to the origin and the supramenton is brought directly below the sellion on the y axis. As a result, the sellion and supramenton are very close across all of the faces, resulting in low variations around the bridge of the nose and the chin. The outer edges of the face have the largest deviations typically. Superimposing the best fit landmarks on the contour plots reveals that the sides of the mask venture into areas with great variation in the front view as shown in Fig. 9a. This also indicates that the landmark points 7-9 were probably chosen to be too wide. A profile view of each face provides another view of the face and more detail about the cheeks of the face which is lacking from the front view. Fig. 9b shows how the best fit mask stays fairly flat on the face and enters areas of substantial variation below the chin and near the eyes.

\section{A)}

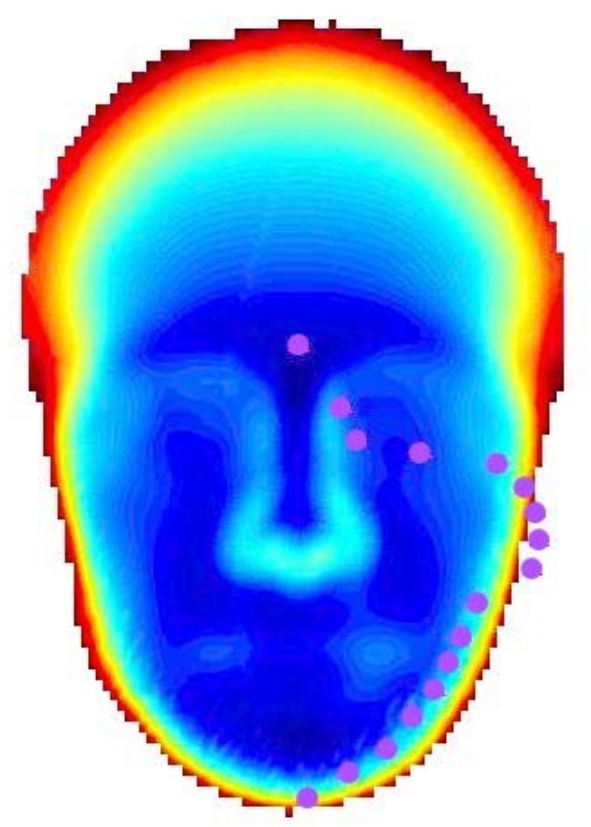

B)

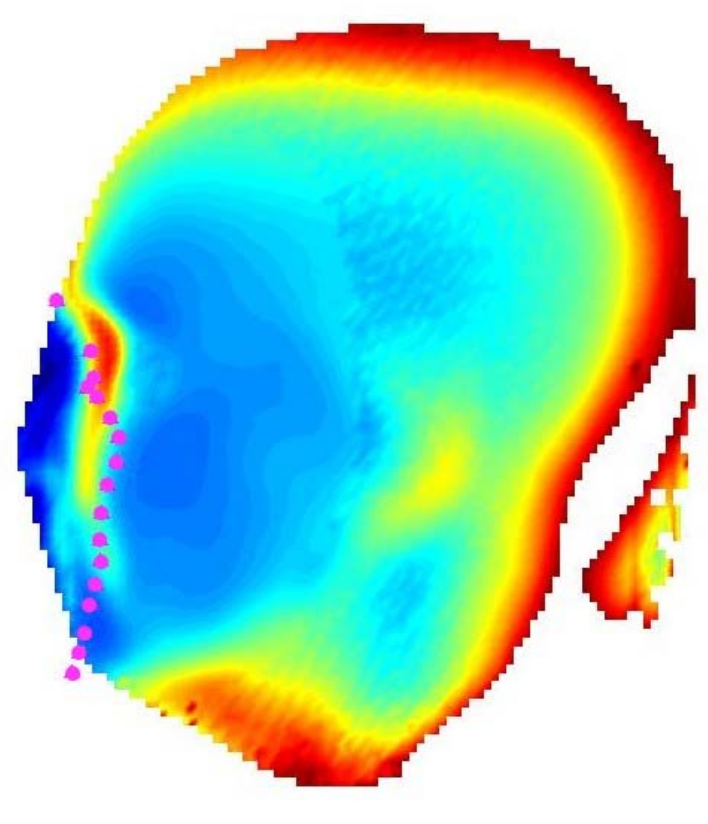

Fig. 9 shows the interpolated contour plot of the standard deviation at each point in the face. The best fit landmarks are shown as magenta circles. Areas of red indicate high deviations and areas of blue are low deviations. Surrounding white areas are deviations in excess of 16 . a) is the front view of the female variation and $b$ ) is the profile view of the female variation. 


\section{CONCLUDING REMARKS}

Perhaps the most surprising result of the experiment is how well the respirator fit the Asian face at all widths when considering the small number of Asians in the U.S. labor force. Granted, these labor statistics may not align precisely with the targeted respirator user as identified by the manufacturer for the medium sized half mask, but it is still significant to note that the Asian face fit well in all categories and the male face fit poorly.

It is also prudent to note that some of the assumptions used in this study may account for some degree of inaccuracy. First of all, the landmark points were chosen in an arbitrary manner by assuming that the minimum $\mathrm{z}$ value for 17 evenly spaced rows would best simulate the contact line for each face. However, the variation analysis proved that some landmarks, particularly 7-9 were impractical and appeared in areas of the face with substantial variation. Moreover, the decision to use landmark points instead of a full surface for the contact area of the mask may have oversimplified the mask. Every face creates a unique area of contact with the respirator; and, by using landmarks this study observes only one of a seemingly infinite number of arrangements. Perhaps a future investigation into the contact area/line between the face and the mask for each individual would identify areas of interest for the face and the mask.

The analysis of the head breadth also offered a glimpse into the difficulty of modeling a fit test using only single dimension measures. Principle Component Analysis, which combines several single dimension measures has shown success in dealing with other areas of the body, but has yet to show a reasonable degree of success in respirator fit tests. Attention to $3 \mathrm{D}$ scans of the face seems necessary if fit testing for respirators is to advance any further.

Unfortunately, rigid body analysis in this study does not take into account any of the malleable features of the face or the mask. In fact, it is not yet certain that a good fit identified in a rigid body analysis even correlates to a good fit in a test panel. Future studies with finite element analysis to model the stretching and deformation in the skin of the face and rubber of the respirator will undoubtedly shed more light on the matter and help 3D anthropometry realize its true potential in fit test modeling. 


\section{REFERENCES}

CAESAR: Civilian American and European Surface Anthropometry Resource web site: http://www.hec.afrl.af.mil/cardlab/CAESAR/index.html.

Coffey, Christopher C., Lawrence, Robert B., Zhuang, Ziqing, Campbell, Donald L., Jensen, Paul A., and Myers, Warren R.: Comparison of five methods for fit-testing N95 filtering-facepiece respirators. App. Occup. Environ. Hyg.: 17:10, 723 - 730 (2002).

Han D.-H., Rhi, J., and Lee J.: Development of prototypes of half-mask facepieces for Koreans using the 3D digitizing design method: a pilot study. Ann. Occup. Hyg.; 48: 707-14 (2004).

Institute of Medicine (IOM). 2007. Assessment of the NIOSH Head-and-Face Anthropometric Survey of U.S. respirator users. Washington, DC: The National Academies Press.

Kim, K., and Kim, H.: Three-dimensional shape analysis of commercial half-facepiece respirators for Koreans: structural approach. J. Int. Soc. Respir. Protect. 23:89 (2006).

Los Alamos Scientific Laboratory of the University of California: Selection of Respirator Test Panels Representative of U.S. Adult Facial Sizes by A.L. Hack, E.C. Hyatt, B.J. Held, et al. (LA5488). Los Alamos, N.M.: Los Alamos Scientific Laboratory of the University of California, 1973.

Oestenstad RK, Dillion HK, Perkin LL. Distribution of faceseal leak sites in a half-mask respirator and their association with facial dimensions. Am Ind Hyg Assoc J. 51: 285290 (1990).

U.S. Bureau of Labor Statistics: Employment situation June 2007. Washington, D.C.: Bureau of Labor Statistics, 2007.

Zhuang, Z., and Bradtmiller, B.: Head-and-face anthropometric survey of U.S. respirator users. J. Occup. Environ. Hyg.; 2: 567-576 (2005). 